Article

\title{
A New Extension of Hardy-Hilbert's Inequality Containing Kernel of Double Power Functions
}

\author{
Bicheng Yang ${ }^{1}$, Shanhe $\mathrm{Wu}^{2, *}$ (1) and Qiang Chen ${ }^{3}$ \\ 1 Institute of Applied Mathematics, Longyan University, Longyan 364012, China; bcyang@gdei.edu.cn \\ 2 Department of Mathematics, Longyan University, Longyan 364012, China \\ 3 Department of Computer Science, Guangdong University of Education, Guangzhou 510303, China; \\ cq_c123@163.com \\ * Correspondence: shanhewu@163.com
}

Received: 8 May 2020; Accepted: 26 May 2020; Published: 2 June 2020

Abstract: In this paper, we provide a new extension of Hardy-Hilbert's inequality with the kernel consisting of double power functions and derive its equivalent forms. The obtained inequalities are then further discussed regarding the equivalent statements of the best possible constant factor related to several parameters. The operator expressions of the extended Hardy-Hilbert's inequality are also considered.

Keywords: Hardy-Hilbert's inequality; best possible constant factor; equivalent statement; operator expression

MSC: 26D15; 26D10; 26A42

\section{Introduction}

The famous Hardy-Hilbert's inequality reads as follows

$$
\sum_{m=1}^{\infty} \sum_{n=1}^{\infty} \frac{a_{m} b_{n}}{m+n}<\frac{\pi}{\sin (\pi / p)}\left(\sum_{m=1}^{\infty} a_{m}^{p}\right)^{\frac{1}{p}}\left(\sum_{n=1}^{\infty} b_{n}^{q}\right)^{\frac{1}{q}}
$$

where $p>1, \frac{1}{p}+\frac{1}{q}=1, a_{m}, b_{n} \geq 0,0<\sum_{m=1}^{\infty} a_{m}^{p}<\infty$ and $0<\sum_{n=1}^{\infty} b_{n}^{q}<\infty$. Furthermore, the constant factor $\frac{\pi}{\sin (\pi / p)}$ in (1) is the best possible (see [1], Theorem 315).

In 2001, Yang [2] used the Beta function,

$$
B(u, v):=\int_{0}^{\infty} \frac{1}{(1+t)^{u+v}} t^{u-1} d t(u, v>0),
$$

to establish an extension of inequality (1) in the case of $p=q=2$, i.e.,

$$
\sum_{m=1}^{\infty} \sum_{n=1}^{\infty} \frac{a_{m} b_{n}}{m+n}<B\left(\frac{\lambda}{2}, \frac{\lambda}{2}\right)\left(\sum_{m=1}^{\infty} m^{1-\lambda} a_{m}^{2} \sum_{n=1}^{\infty} n^{1-\lambda} b_{n}^{2}\right)^{\frac{1}{2}},
$$

Motivated by the result of Yang, in 2006, Krnić and Pečarić [3] proposed a further extension of inequality (1) by introducing parameters $\lambda_{1}$ and $\lambda_{2}$, as follows

$$
\sum_{m=1}^{\infty} \sum_{n=1}^{\infty} \frac{a_{m} b_{n}}{(m+n)^{\lambda}}<B\left(\lambda_{1}, \lambda_{2}\right)\left[\sum_{m=1}^{\infty} m^{p\left(1-\lambda_{1}\right)-1} a_{m}^{p}\right]^{\frac{1}{p}}\left[\sum_{n=1}^{\infty} n^{q\left(1-\lambda_{2}\right)-1} b_{n}^{q}\right]^{\frac{1}{q}}
$$


where $\lambda_{i} \in(0,2](i=1,2), \lambda_{1}+\lambda_{2}=\lambda \in(0,4]$, the constant factor $B\left(\lambda_{1}, \lambda_{2}\right)$ in (4) is the best possible.

For $\lambda=1, \lambda_{1}=\frac{1}{q}, \lambda_{2}=\frac{1}{p}$, inequality (4) reduces to the Hardy-Hilbert's inequality (1); for $p=q=2, \lambda_{1}=\lambda_{2}=\frac{\lambda}{2}$, inequality (4) reduces to the Yang's inequality (3).

Recently, with the help of inequality (4), Adiyasuren, Batbold and Azar [4] established a new Hilbert-type inequality containing the kernel $\frac{1}{(m+n)^{\lambda}}$ and gave the best possible constant factor $\lambda_{1} \lambda_{2} B\left(\lambda_{1}, \lambda_{2}\right)$ involving partial sums, as follows:

If $\lambda_{i} \in(0,1] \cap(0, \lambda)(i=1,2), \lambda_{1}+\lambda_{2}=\lambda \in(0,2]$, then

$$
\sum_{m=1}^{\infty} \sum_{n=1}^{\infty} \frac{a_{m} b_{n}}{(m+n)^{\lambda}}<\lambda_{1} \lambda_{2} B\left(\lambda_{1}, \lambda_{2}\right)\left(\sum_{m=1}^{\infty} m^{-p \lambda_{1}-1} A_{m}^{p}\right)^{\frac{1}{p}}\left(\sum_{n=1}^{\infty} n^{-q \lambda_{2}-1} B_{n}^{q}\right)^{\frac{1}{q}},
$$

where, for $a_{m}, b_{n} \geq 0$, the partial sums $A_{m}=\sum_{i=1}^{m} a_{i}$ and $B_{n}=\sum_{k=1}^{n} b_{k}$ satisfy

$$
0<\sum_{m=1}^{\infty} m^{-p \lambda_{1}-1} A_{m}^{p}<\infty \text { and } 0<\sum_{n=1}^{\infty} n^{-q \lambda_{2}-1} B_{n}^{q}<\infty .
$$

It is well-known that Hardy-Hilbert's inequality (1) and its extensions have important applications in real analysis and operator theory (see [5-15]).

Apart from the inequalities of Hardy-Hilbert type mentioned above, the following half-discrete Hardy-Hilbert inequality is also attractive (see [1], Theorem 351), i.e.,

If $K(t)(t>0)$ is a decreasing function, $p>1, \frac{1}{p}+\frac{1}{q}=1,0<\varphi(s)=\int_{0}^{\infty} K(t) t^{s-1} d t<\infty, a_{n} \geq 0$, $0<\sum_{n=1}^{\infty} a_{n}^{p}<\infty$, then

$$
\int_{0}^{\infty} x^{p-2}\left(\sum_{n=1}^{\infty} K(n x) a_{n}\right)^{p} d x<\varphi^{p}\left(\frac{1}{q}\right) \sum_{n=1}^{\infty} a_{n}^{p} .
$$

A lot of investigations have been given to the extensions of inequality (6) and its applications, see [16-22] and references cited therein.

Recently, it has come to our attention that some results were provided by Hong and Wen in [23]: in the paper they studied the equivalent statements of the extended inequalities (1) and (2), and estimated the best possible constant factor for several parameters. Inspired by the ideas of Hong and Wen in [23], using the Euler-Maclaurin summation formula, Yang, Wu and Chen [24] presented an extension of Hardy-Littlewood-Polya's inequality involving the kernel $\frac{1}{(\max \{m, n\})^{\lambda}}$ as follows

$$
\begin{gathered}
\sum_{n=1}^{\infty} \sum_{m=1}^{\infty} \frac{a_{m} b_{n}}{(\max \{m, n\})^{\lambda}}<k_{\lambda}^{\frac{1}{p}}\left(\lambda_{2}\right) k_{\lambda}^{\frac{1}{q}}\left(\lambda_{1}\right) \\
\times\left\{\sum_{m=1}^{\infty} m^{p\left[1-\left(\frac{\lambda-\lambda_{2}}{p}+\frac{\lambda_{1}}{q}\right)\right]-1} a_{m}^{p}\right\}^{\frac{1}{p}}\left\{\sum_{n=1}^{\infty} n^{q\left[1-\left(\frac{\lambda_{2}}{p}+\frac{\lambda-\lambda_{1}}{q}\right)\right]-1} b_{n}^{q}\right\}^{\frac{1}{q}} .
\end{gathered}
$$

where $p>1, \frac{1}{p}+\frac{1}{q}=1, \lambda \in(0,3], \lambda_{i} \in\left(0, \frac{11}{8}\right] \cap(0, \lambda), k_{\lambda}\left(\lambda_{i}\right)=\frac{\lambda}{\lambda_{i}\left(\lambda-\lambda_{i}\right)}(i=1,2), a_{m}, b_{n} \geq 0$.

Yang, Wu and Wang [25] established a Hilbert-type inequality containing the positive homogeneous kernel $(\min \{m, n\})^{\lambda}$, i.e.,

$$
\begin{gathered}
\sum_{n=1}^{\infty} \sum_{m=1}^{\infty}(\min \{m, n\})^{\lambda} a_{m} b_{n}<k_{\lambda}^{\frac{1}{p}}\left(\lambda_{1}\right) k_{\lambda}^{\frac{1}{q}}\left(\lambda_{2}\right) \\
\times\left[\sum_{m=1}^{\infty} m^{p\left(1+\frac{\lambda_{1}}{p}+\frac{\lambda-\lambda_{2}}{q}\right)-1} a_{m}^{p}\right]^{\frac{1}{p}}\left[\sum_{n=1}^{\infty} n^{q\left(1+\frac{\lambda_{2}}{q}+\frac{\lambda-\lambda_{1}}{p}\right)-1} b_{n}^{q}\right]^{\frac{1}{q}},
\end{gathered}
$$

where $p>1, \frac{1}{p}+\frac{1}{q}=1, \lambda \in\left(0, \frac{34}{11}\right], \lambda_{i} \in\left(0, \frac{11}{8}\right] \cap(0, \lambda), k_{\lambda}\left(\lambda_{i}\right)=\frac{\lambda}{\lambda_{i}\left(\lambda-\lambda_{i}\right)}(i=1,2), a_{m}, b_{n} \geq 0$. 
Yang, Wu and Liao [26] gave an extension of Hardy-Hilbert's inequality involving the kernel $\frac{1}{m^{\lambda}+n^{\lambda}}$, that is,

$$
\begin{gathered}
\sum_{n=1}^{\infty} \sum_{m=1}^{\infty} \frac{a_{m} b_{n}}{m^{\lambda}+n^{\lambda}}<k_{\lambda}^{\frac{1}{p}}\left(\lambda_{2}\right) k_{\lambda}^{\frac{1}{q}}\left(\lambda_{1}\right) \\
\times\left\{\sum_{m=1}^{\infty} m^{p\left[1-\left(\frac{\lambda-\lambda_{2}}{p}+\frac{\lambda_{1}}{q}\right)\right]-1} a_{m}^{p}\right\}^{\frac{1}{p}}\left\{\sum_{n=1}^{\infty} n^{q\left[1-\left(\frac{\lambda_{2}}{p}+\frac{\lambda-\lambda_{1}}{q}\right)\right]-1} b_{n}^{q}\right\}^{\frac{1}{q}},
\end{gathered}
$$

where $p>1, \frac{1}{p}+\frac{1}{q}=1, \lambda \in\left(0, \frac{5}{2}\right], \lambda_{i} \in\left(0, \frac{5}{4}\right] \cap(0, \lambda), k_{\lambda}\left(\lambda_{i}\right)=\frac{\pi}{\lambda \sin \left(\pi \lambda_{i} / \lambda\right)}(i=1,2), a_{m}, b_{n} \geq 0$.

This paper continues the studies from [24-26]; we establish a new extension of Hardy-Hilbert's inequality with the kernel consisting of double power function

$$
\frac{1}{\left(m^{\alpha}+n^{\beta}\right)^{\lambda}}(\lambda \in(0,6], \alpha, \beta \in(0,1]) .
$$

Based on the obtained inequality, we derive its equivalent form and discuss the equivalent statements of the best possible constant factor related to several parameters. The operator expressions and some particular cases of the obtained inequality of Hardy-Hilbert type are also considered.

\section{Some Lemmas}

In what follows, we suppose that $p>1, \frac{1}{p}+\frac{1}{q}=1, \mathrm{~N}=\{1,2, \cdots\}, \lambda \in(0,6], \alpha, \beta \in(0,1]$, $\lambda_{1} \in\left(0, \frac{2}{\alpha}\right] \cap(0, \lambda), \lambda_{2} \in\left(0, \frac{2}{\beta}\right] \cap(0, \lambda), k_{\lambda}\left(\lambda_{i}\right):=B\left(\lambda_{i}, \lambda-\lambda_{i}\right)(i=1,2)$. We also suppose that $a_{m}, b_{n} \geq 0$ are such that

$$
0<\sum_{m=1}^{\infty} m^{p\left[1-\alpha\left(\frac{\lambda-\lambda_{2}}{p}+\frac{\lambda_{1}}{q}\right)\right]-1} a_{m}^{p}<\infty \text { and } 0<\sum_{n=1}^{\infty} n^{q\left[1-\beta\left(\frac{\lambda-\lambda_{1}}{q}+\frac{\lambda_{2}}{p}\right)\right]-1} b_{n}^{q}<\infty .
$$

Lemma 1. For $\lambda \in(0,6], \alpha, \beta \in(0,1], \lambda_{2} \in\left(0, \frac{2}{\beta}\right] \cap(0, \lambda)$, define the following weight coefficient:

$$
\omega\left(\lambda_{2}, m\right):=m^{\alpha\left(\lambda-\lambda_{2}\right)} \sum_{n=1}^{\infty} \frac{\beta n^{\beta \lambda_{2}-1}}{\left(m^{\alpha}+n^{\beta}\right)^{\lambda}}(m \in \mathrm{N}) .
$$

Then we have the following inequalities

$$
0<k_{\lambda}\left(\lambda_{2}\right)\left(1-O\left(\frac{1}{m^{\alpha \lambda_{2}}}\right)\right)<\omega\left(\lambda_{2}, m\right)<k_{\lambda}\left(\lambda_{2}\right)(m \in \mathrm{N}),
$$

where $O\left(\frac{1}{m^{\alpha \lambda_{2}}}\right):=\frac{1}{k_{\lambda}\left(\lambda_{2}\right)} \int_{0}^{\frac{1}{m^{\alpha}}} \frac{u^{\lambda_{2}-1}}{(1+u)^{\lambda}} d u>0$.

Proof. For fixed $m \in \mathrm{N}$, we define the function $g(m, t)$ as follows:

$$
g(m, t):=\frac{\beta t^{\beta \lambda_{2}-1}}{\left(m^{\alpha}+t^{\beta}\right)^{\lambda}}(t>0) .
$$

By using the Euler-Maclaurin summation formula (see [2,3]), for the Bernoulli function of 1-order $P_{1}(t):=t-[t]-\frac{1}{2}$, we have

$$
\begin{aligned}
\sum_{n=1}^{\infty} g(m, n) & =\int_{1}^{\infty} g(m, t) d t+\frac{1}{2} g(m, 1)+\int_{1}^{\infty} P_{1}(t) g^{\prime}(m, t) d t \\
& =\int_{0}^{\infty} g(m, t) d t-h(m), \\
h(m): & =\int_{0}^{1} g(m, t) d t-\frac{1}{2} g(m, 1)-\int_{1}^{\infty} P_{1}(t) g^{\prime}(m, t) d t .
\end{aligned}
$$


We obtain $-\frac{1}{2} g(m, 1)=\frac{-\beta}{2\left(m^{\alpha}+1\right)^{\lambda}}$, and

$$
\begin{aligned}
& -g^{\prime}(m, t)=-\frac{\beta\left(\beta \lambda_{2}-1\right) t^{\beta \lambda_{2}-2}}{\left(m^{\alpha}+t^{\beta}\right)^{\lambda}}+\frac{\beta^{2} \lambda t^{\beta+\beta \lambda_{2}-2}}{\left(m^{\alpha}+t^{\beta}\right)^{\lambda+1}} \\
& =-\frac{\beta\left(\beta \lambda_{2}-1\right) t^{\beta \lambda_{2}-2}}{\left(m^{\alpha}+t^{\beta}\right)^{\lambda}}+\frac{\beta^{2} \lambda\left(m^{\alpha}+t^{\beta}-m^{\alpha}\right) t^{\beta \lambda_{2}-2}}{\left(m^{\alpha}+t^{\beta}\right)^{\lambda+1}} \\
& =\frac{\beta\left(\beta \lambda-\beta \lambda_{2}+1\right) t^{\beta \lambda_{2}-2}}{\left(m^{\alpha}+t^{\beta}\right)^{\lambda}}-\frac{\beta^{2} \lambda m^{\alpha} t^{\beta \lambda_{2}-2}}{\left(m^{\alpha}+t^{\beta}\right)^{\lambda+1}} .
\end{aligned}
$$

Integrating by parts, we have

$$
\begin{aligned}
& \int_{0}^{1} g(m, t) d t=\int_{0}^{1} \frac{\beta t^{\beta \lambda_{2}-1}}{\left(m^{\alpha}+t^{\beta}\right)^{\lambda}} d t \stackrel{u=t^{\beta}}{=} \int_{0}^{1} \frac{u^{\lambda_{2}-1}}{\left(m^{\alpha}+u\right)^{\lambda}} d u \\
& =\frac{1}{\lambda_{2}} \int_{0}^{1} \frac{d u^{\lambda_{2}}}{\left(m^{\alpha}+u\right)^{\lambda}}=\left.\frac{1}{\lambda_{2}} \frac{u^{\lambda_{2}}}{\left(m^{\alpha}+u\right)^{\lambda}}\right|_{0} ^{1}+\frac{\lambda}{\lambda_{2}} \int_{0}^{1} \frac{u^{\lambda_{2}}}{\left(m^{\alpha}+u\right)^{\lambda+1}} d u \\
& =\frac{1}{\lambda_{2}} \frac{1}{\left(m^{\alpha}+1\right)^{\lambda}}+\frac{\lambda}{\lambda_{2}\left(\lambda_{2}+1\right)} \int_{0}^{1} \frac{d u^{\lambda_{2}+1}}{\left(m^{\alpha}+u\right)^{\lambda+1}} \\
& >\frac{1}{\lambda_{2}} \frac{1}{\left(m^{\alpha}+1\right)^{\lambda}}+\frac{\lambda}{\lambda_{2}\left(\lambda_{2}+1\right)}\left[\frac{u^{\lambda_{2}+1}}{\left(m^{\alpha}+u\right)^{\lambda+1}}\right]_{0}^{1}+\frac{\lambda(\lambda+1)}{\lambda_{2}\left(\lambda_{2}+1\right)\left(m^{\alpha}+1\right)^{\lambda+2}} \int_{0}^{1} u^{\lambda_{2}+1} d u \\
& =\frac{1}{\lambda_{2}} \frac{1}{\left(m^{\alpha}+1\right)^{\lambda}}+\frac{\lambda}{\lambda_{2}\left(\lambda_{2}+1\right)} \frac{1}{\left(m^{\alpha}+1\right)^{\lambda+1}}+\frac{\lambda(\lambda+1)}{\lambda_{2}\left(\lambda_{2}+1\right)\left(\lambda_{2}+2\right)} \frac{1}{\left(m^{\alpha}+1\right)^{\lambda+2}} .
\end{aligned}
$$

For $0<\lambda_{2} \leq \frac{2}{\beta}, 0<\beta \leq 1, \lambda_{2}<\lambda \leq 6$, it follows that

$$
(-1)^{i} \frac{d^{i}}{d t^{i}}\left[\frac{t^{\beta \lambda_{2}-2}}{\left(m^{\alpha}+t^{\beta}\right)^{\lambda}}\right]>0,(-1)^{i} \frac{d^{i}}{d t^{i}}\left[\frac{t^{\beta \lambda_{2}-2}}{\left(m^{\alpha}+t^{\beta}\right)^{\lambda+1}}\right]>0(i=0,1,2,3) .
$$

Utilizing the Euler-Maclaurin summation formula (see [2,3]), we obtain

$$
\begin{gathered}
\quad \beta\left(\beta \lambda-\beta \lambda_{2}+1\right) \int_{1}^{\infty} P_{1}(t) \frac{t^{\beta \lambda_{2}-2}}{\left(m^{\alpha}+t^{\beta}\right)^{\lambda}} d t>-\frac{\beta\left(\beta \lambda-\beta \lambda_{2}+1\right)}{12\left(m^{\alpha}+1\right)^{\lambda}}, \\
-\beta^{2} m^{\alpha} \lambda \int_{1}^{\infty} P_{1}(t) \frac{t^{\beta \lambda_{2}-2}}{\left(m^{\alpha}+t^{\beta}\right)^{\lambda+1}} d t>\frac{\beta^{2} m^{\alpha} \lambda}{12\left(m^{\alpha}+1\right)^{\lambda+1}}-\frac{\beta^{2} m^{\alpha} \lambda}{720}\left[\frac{t^{\beta \lambda_{2}-2}}{\left(m^{\alpha}+t^{\beta}\right)^{\lambda+1}}\right]_{{ }^{\prime \prime}}^{t=1} \\
>\frac{\beta^{2}\left(m^{\alpha}+1-1\right) \lambda}{12\left(m^{\alpha}+1\right)^{\lambda+1}}-\frac{\beta^{2}\left(m^{\alpha}+1\right) \lambda}{720}\left[\frac{(\lambda+1)(\lambda+2) \beta^{2}}{\left(m^{\alpha}+1\right)^{\lambda+3}}+\frac{\beta(\lambda+1)\left(5-\beta-2 \beta \lambda_{2}\right)}{\left(m^{\alpha}+1\right)^{\lambda+2}}+\frac{\left(2-\beta \lambda_{2}\right)\left(3-\beta \lambda_{2}\right)}{\left(m^{\alpha}+1\right)^{\lambda+1}}\right] \\
=\frac{\beta^{2} \lambda}{12\left(m^{\alpha}+1\right)^{\lambda}}-\frac{\beta^{2} \lambda}{12\left(m^{\alpha}+1\right)^{\lambda+1}}-\frac{\beta^{2} \lambda}{720}\left[\frac{(\lambda+1)(\lambda+2) \beta^{2}}{\left(m^{\alpha}+1\right)^{\lambda+2}}+\frac{\beta(\lambda+1)\left(5-\beta-2 \beta \lambda_{2}\right)}{\left(m^{\alpha}+1\right)^{\lambda+1}}+\frac{\left(2-\beta \lambda_{2}\right)\left(3-\beta \lambda_{2}\right)}{\left(m^{\alpha}+1\right)^{\lambda}}\right] .
\end{gathered}
$$

and then, one has

$$
\begin{gathered}
h(m)>\frac{1}{\left(m^{\alpha}+1\right)^{\lambda}} h_{1}+\frac{\lambda}{\left(m^{\alpha}+1\right)^{\lambda+1}} h_{2}+\frac{\lambda(\lambda+1)}{\left(m^{\alpha}+1\right)^{\lambda+2}} h_{3}, h_{1}:=\frac{1}{\lambda_{2}}-\frac{\beta}{2}-\frac{\beta-\beta^{2} \lambda_{2}}{12}-\frac{\beta^{2} \lambda\left(2-\beta \lambda_{2}\right)\left(3-\beta \lambda_{2}\right)}{720}, \\
h_{2}:=\frac{1}{\lambda_{2}\left(\lambda_{2}+1\right)}-\frac{\beta^{2}}{12}-\frac{\beta^{3}(\lambda+1)\left(5-\beta-2 \beta \lambda_{2}\right)}{720}
\end{gathered}
$$

and

$$
h_{3}:=\frac{1}{\lambda_{2}\left(\lambda_{2}+1\right)\left(\lambda_{2}+2\right)}-\frac{\beta^{4}(\lambda+2)}{720} .
$$

Further, we deduce that

$$
h_{1} \geq \frac{1}{\lambda_{2}}-\frac{\beta}{2}-\frac{\beta-\beta^{2} \lambda_{2}}{12}-\frac{\lambda \beta^{2}\left(2-\beta \lambda_{2}\right)\left(3-\beta \lambda_{2}\right)}{720}=\frac{g\left(\lambda_{2}\right)}{720 \lambda_{2}}
$$

where the function $g(\sigma)\left(\sigma \in\left(0, \frac{2}{\beta}\right]\right)$ is defined by

$$
g(\sigma):=720-\left(420 \beta+6 \lambda \beta^{2}\right) \sigma+\left(60 \beta^{2}+5 \lambda \beta^{3}\right) \sigma^{2}-\lambda \beta^{4} \sigma^{3} .
$$


For $\beta \in(0,1], \lambda \in(0,6]$ and $\sigma \in\left(0, \frac{2}{\beta}\right]$, we have

$$
\begin{aligned}
g^{\prime}(\sigma) & =-\left(420 \beta+6 \lambda \beta^{2}\right)+2\left(60 \beta^{2}+5 \lambda \beta^{3}\right) \sigma-3 \beta^{4} \sigma^{2} \\
& \leq-420 \beta-6 \lambda \beta^{2}+2\left(60 \beta^{2}+5 \lambda \beta^{3}\right) \frac{\beta}{2} \\
& =(14 \lambda \beta-180) \beta<0
\end{aligned}
$$

Thus, it follows that

$$
h_{1} \geq \frac{g\left(\lambda_{2}\right)}{720 \lambda_{2}} \geq \frac{g(2 / \beta)}{720 \lambda_{2}}=\frac{1}{6 \lambda_{2}}>0 .
$$

We obtain that for $\lambda_{2} \in\left(0, \frac{2}{\beta}\right]$,

$$
h_{2}>\frac{\beta^{2}}{6}-\frac{\beta^{2}}{12}-\frac{5(\lambda+1) \beta^{2}}{720}=\left(\frac{1}{12}-\frac{\lambda+1}{140}\right) \beta^{2}>0(0<\lambda \leq 6)
$$

and

$$
h_{3} \geq\left(\frac{1}{24}-\frac{\lambda+2}{720}\right) \beta^{3}>0(0<\lambda \leq 6) .
$$

Hence, we get $h(m)>0$. Setting $t=m^{\alpha / \beta} u^{1 / \beta}$, it follows that

$$
\begin{aligned}
\omega\left(\lambda_{2}, m\right) & =m^{\alpha\left(\lambda-\lambda_{2}\right)} \sum_{n=1}^{\infty} g(m, n)<m^{\alpha\left(\lambda-\lambda_{2}\right)} \int_{0}^{\infty} g(m, t) d t \\
& =m^{\alpha\left(\lambda-\lambda_{2}\right)} \int_{0}^{\infty} \frac{\beta t^{\beta} \lambda_{2}-1}{\left(m^{\alpha}+t^{\beta}\right)^{\lambda}} d t=\int_{0}^{\infty} \frac{u^{\lambda_{2}-1}}{(1+u)^{\lambda}} d u=B\left(\lambda_{2}, \lambda-\lambda_{2}\right) .
\end{aligned}
$$

On the other hand, we also have

$$
\begin{aligned}
\sum_{n=1}^{\infty} g(m, n) & =\int_{1}^{\infty} g(m, t) d t+\frac{1}{2} g(m, 1)+\int_{1}^{\infty} P_{1}(t) g^{\prime}(m, t) d t \\
& =\int_{1}^{\infty} g(m, t) d t+H(m), \\
H(m) & :=\frac{1}{2} g(m, 1)+\int_{1}^{\infty} P_{1}(t) g^{\prime}(m, t) d t .
\end{aligned}
$$

Note that $\frac{1}{2} g(m, 1)=\frac{\beta}{2\left(m^{\alpha}+1\right)^{\lambda}}$ and

$$
g^{\prime}(m, t)=-\frac{\beta\left(\beta \lambda-\beta \lambda_{2}+1\right) t^{\beta \lambda_{2}-2}}{\left(m^{\alpha}+t^{\beta}\right)^{\lambda}}+\frac{\beta^{2} \lambda m^{\alpha} t^{\beta \lambda_{2}-2}}{\left(m^{\alpha}+t^{\beta}\right)^{\lambda+1}} .
$$

For $\lambda_{2} \in\left(0, \frac{2}{\beta}\right] \cap(0, \lambda), 0<\lambda \leq 6$, by using the Euler-Maclaurin summation formula (see $[2,3]$ ), we obtain

$$
-\beta\left(\beta \lambda-\beta \lambda_{2}+1\right) \int_{1}^{\infty} P_{1}(t) \frac{t^{\beta \lambda_{2}-2}}{\left(m^{\alpha}+t^{\beta}\right)^{\lambda}} d t>0
$$

and

$$
\beta^{2} m^{\alpha} \lambda \int_{1}^{\infty} P_{1}(t) \frac{t^{\beta \lambda_{2}-2}}{\left(m^{\alpha}+t^{\beta}\right)^{\lambda+1}} d t>-\frac{\beta^{2} m^{\alpha} \lambda}{12\left(m^{\alpha}+1\right)^{\lambda+1}}>-\frac{\beta^{2} \lambda}{12\left(m^{\alpha}+1\right)^{\lambda}} .
$$

Hence, we have

$$
H(m)>\frac{\beta}{2\left(m^{\alpha}+1\right)^{\lambda}}-\frac{\beta^{2} \lambda}{12\left(m^{\alpha}+1\right)^{\lambda}} \geq \frac{\beta}{2\left(m^{\alpha}+1\right)^{\lambda}}-\frac{6 \beta}{12\left(m^{\alpha}+1\right)^{\lambda}}=0 .
$$


Further, it follows that

$$
\begin{aligned}
\omega\left(\lambda_{2}, m\right) & =m^{\alpha\left(\lambda-\lambda_{2}\right)} \sum_{n=1}^{\infty} g(m, n)>m^{\alpha\left(\lambda-\lambda_{2}\right)} \int_{1}^{\infty} g(m, t) d t \\
& =m^{\alpha\left(\lambda-\lambda_{2}\right)} \int_{0}^{\infty} g(m, t) d t-m^{\alpha\left(\lambda-\lambda_{2}\right)} \int_{0}^{1} g(m, t) d t \\
& =k_{\lambda}\left(\lambda_{2}\right)\left[1-\frac{1}{k_{\lambda}\left(\lambda_{2}\right)} \int_{0}^{\frac{1}{m^{\alpha}}} \frac{u^{\lambda_{2}-1}}{(1+u)^{\lambda}} d u\right]>0,
\end{aligned}
$$

where we set $O\left(\frac{1}{m^{\alpha \lambda_{2}}}\right)=\frac{1}{k_{\lambda}\left(\lambda_{2}\right)} \int_{0}^{\frac{1}{m^{\alpha}}} \frac{u^{\lambda_{2}-1}}{(1+u)^{\lambda}} d u>0$ satisfying

$$
0<\int_{0}^{\frac{1}{m^{\alpha}}} \frac{u^{\lambda_{2}-1}}{(1+u)^{\lambda}} d u<\int_{0}^{\frac{1}{m^{\alpha}}} u^{\lambda_{2}-1} d u=\frac{1}{\lambda_{2} m^{\alpha \lambda_{2}}} .
$$

Therefore, we obtain inequalities (12). The Lemma 1 is proved.

Lemma 2. The following extended Hardy-Hilbert's inequality holds true:

$$
\begin{gathered}
I=\sum_{n=1}^{\infty} \sum_{m=1}^{\infty} \frac{a_{m} b_{n}}{\left(m^{\alpha}+n^{\beta}\right)^{\lambda}}<\left(\frac{1}{\beta} k_{\lambda}\left(\lambda_{2}\right)\right)^{\frac{1}{p}}\left(\frac{1}{\alpha} k_{\lambda}\left(\lambda_{1}\right)\right)^{\frac{1}{q}} \\
\times\left\{\sum_{m=1}^{\infty} m^{p\left[1-\alpha\left(\frac{\lambda-\lambda_{2}}{p}+\frac{\lambda_{1}}{q}\right)\right]-1} a_{m}^{p}\right\}^{\frac{1}{p}}\left\{\sum_{n=1}^{\infty} n^{q\left[1-\beta\left(\frac{\lambda-\lambda_{1}}{q}+\frac{\lambda_{2}}{p}\right)\right]-1} b_{n}^{q}\right\}^{\frac{1}{q}}
\end{gathered}
$$

Proof. Following the way of the proof of Lemma 1, for $n \in \mathbf{N}$, we have the following inequality

$$
0<k_{\lambda}\left(\lambda_{1}\right)\left(1-O\left(\frac{1}{n^{\beta \lambda_{1}}}\right)\right)<\omega\left(\lambda_{1}, n\right):=n^{\beta\left(\lambda-\lambda_{1}\right)} \sum_{m=1}^{\infty} \frac{\alpha m^{\alpha \lambda_{1}-1}}{\left(m^{\alpha}+n^{\beta}\right)^{\lambda}}<k_{\lambda}\left(\lambda_{1}\right) .
$$

Using Hölder's inequality (see [27]), we obtain

$$
\begin{aligned}
I & =\sum_{n=1}^{\infty} \sum_{m=1}^{\infty} \frac{1}{\left(m^{\alpha}+n^{\beta}\right)^{\lambda}}\left[\frac{m^{\alpha\left(1-\lambda_{1}\right) / q}\left(\beta n^{\beta-1}\right)^{1 / p}}{n^{\beta\left(1-\lambda_{2}\right) / p}\left(\alpha m^{\alpha-1}\right)^{1 / q}} a_{m}\right]\left[\frac{n^{\beta\left(1-\lambda_{2}\right) / p}\left(\alpha m^{\alpha-1}\right)^{1 / q}}{m^{\alpha\left(1-\lambda_{1}\right) / q}\left(\beta n^{\beta-1}\right)^{1 / p}} b_{n}\right] \\
& \leq\left[\sum_{m=1}^{\infty} \sum_{n=1}^{\infty} \frac{\beta}{\left(m^{\alpha}+n^{\beta}\right)^{\lambda}} \frac{m^{\alpha\left(1-\lambda_{1}\right)(p-1)} n^{\beta-1} a_{m}^{p}}{n^{\beta\left(1-\lambda_{2}\right)}\left(\alpha m^{\alpha-1}\right)^{p-1}}\right]^{\frac{1}{p}}\left[\sum_{n=1}^{\infty} \sum_{m=1}^{\infty} \frac{\alpha}{\left(m^{\alpha}+n^{\beta}\right)^{\lambda}} \frac{n^{\beta\left(1-\lambda_{2}\right)(q-1)} m^{\alpha-1} b_{n}^{q}}{m^{\alpha\left(1-\lambda_{1}\right)}\left(\beta n^{\beta-1}\right)^{q-1}}\right]^{\frac{1}{q}} \\
& =\frac{1}{\alpha^{1 / q} \beta^{1 / p}}\left\{\sum_{m=1}^{\infty} \omega\left(\lambda_{2}, m\right) m^{p\left[1-\alpha\left(\frac{\lambda-\lambda_{2}}{p}+\frac{\lambda_{1}}{q}\right)\right]-1} a_{m}^{p}\right\}^{\frac{1}{p}} \\
& \times\left\{\sum_{n=1}^{\infty} \omega\left(\lambda_{1}, n\right) n^{q\left[1-\beta\left(\frac{\lambda-\lambda_{1}}{q}+\frac{\lambda_{2}}{p}\right)\right]-1} b_{n}^{q}\right\}^{\frac{1}{q}} .
\end{aligned}
$$

Thus, from inequalities (12) and (14), we obtain (13). The proof of Lemma 2 is complete.

Remark 1. For $\lambda_{1}+\lambda_{2}=\lambda \in(0,6]$, setting

$$
K_{\lambda}\left(\lambda_{1}\right):=\frac{1}{\alpha^{1 / q} \beta^{1 / p}} B\left(\lambda_{1}, \lambda_{2}\right)
$$

By inequality (10), we obtain

$$
\begin{gathered}
\omega\left(\lambda_{1}, n\right)=n^{\beta \lambda_{2}} \sum_{m=1}^{\infty} \frac{\alpha m^{\alpha \lambda_{1}-1}}{\left(m^{\alpha}+n^{\beta}\right)^{\lambda}} \\
0<\sum_{m=1}^{\infty} m^{p\left(1-\alpha \lambda_{1}\right)-1} a_{m}^{p}<\infty \text { and } 0<\sum_{n=1}^{\infty} n^{q\left(1-\beta \lambda_{2}\right)-1} b_{n}^{q}<\infty,
\end{gathered}
$$


and the following inequality

$$
\sum_{n=1}^{\infty} \sum_{m=1}^{\infty} \frac{a_{m} b_{n}}{\left(m^{\alpha}+n^{\beta}\right)^{\lambda}}<K_{\lambda}\left(\lambda_{1}\right)\left[\sum_{m=1}^{\infty} m^{p\left(1-\alpha \lambda_{1}\right)-1} a_{m}^{p}\right]^{\frac{1}{p}}\left[\sum_{n=1}^{\infty} n^{q\left(1-\beta \lambda_{2}\right)-1} b_{n}^{q}\right]^{\frac{1}{q}} .
$$

Lemma 3. For $\lambda_{1}+\lambda_{2}=\lambda \in(0,6]$, the constant factor $K_{\lambda}\left(\lambda_{1}\right)$ in (15) is the best possible.

Proof. For any $0<\varepsilon<p \lambda_{1}$, we set

$$
\widetilde{a}_{m}:=m^{\alpha\left(\lambda_{1}-\frac{\varepsilon}{p}\right)-1}, \widetilde{b}_{n}:=n^{\beta\left(\lambda_{2}-\frac{\varepsilon}{q}\right)-1}(m, n \in \mathrm{N}) .
$$

If there exists a constant $M \leq K_{\lambda}\left(\lambda_{1}\right)$ such that (15) is valid when replacing $K_{\lambda}\left(\lambda_{1}\right)$ by $M$, then in particular, by substitution of $a_{m}=\widetilde{a}_{m}$ and $b_{n}=\widetilde{b}_{n}$ in (13), we have

$$
\widetilde{I}:=\sum_{n=1}^{\infty} \sum_{m=1}^{\infty} \frac{\widetilde{a}_{m} \widetilde{b}_{n}}{\left(m^{\alpha}+n^{\beta}\right)^{\lambda}}<M\left[\sum_{m=1}^{\infty} m^{p\left(1-\alpha \lambda_{1}\right)-1} \widetilde{a}_{m}^{p}\right]^{\frac{1}{p}}\left[\sum_{n=1}^{\infty} n^{q\left(1-\beta \lambda_{2}\right)-1} \widetilde{b}_{n}^{q}\right]^{\frac{1}{q}} .
$$

In the following, we shall prove that $M \geq K_{\lambda}\left(\lambda_{1}\right)$, and this will lead to $M=K_{\lambda}\left(\lambda_{1}\right)$ is the best possible constant factor in (16).

By inequality (16) and the decreasingness property of series, we obtain

$$
\begin{aligned}
\widetilde{I} & <M\left[\sum_{m=1}^{\infty} m^{p\left(1-\alpha \lambda_{1}\right)-1} m^{p \alpha \lambda_{1}-\alpha \varepsilon-p}\right]^{\frac{1}{p}}\left[\sum_{n=1}^{\infty} n^{q\left(1-\beta \lambda_{2}\right)-1} n^{q \beta \lambda_{2}-\beta \varepsilon-q}\right]^{\frac{1}{q}} \\
& =M\left(1+\sum_{m=2}^{\infty} m^{-\alpha \varepsilon-1}\right)^{\frac{1}{p}}\left(1+\sum_{n=2}^{\infty} n^{-\beta \varepsilon-1}\right)^{\frac{1}{q}} \\
& <M\left(1+\int_{1}^{\infty} x^{-\alpha \varepsilon-1} d x\right)^{\frac{1}{p}}\left(1+\int_{1}^{\infty} y^{-\beta \varepsilon-1} d y\right)^{\frac{1}{q}} \\
& =\frac{M}{\varepsilon}\left(\frac{1}{\alpha}+\varepsilon\right)^{\frac{1}{p}}\left(\frac{1}{\beta}+\varepsilon\right)^{\frac{1}{q}}
\end{aligned}
$$

By using inequality (14) and setting

$$
\hat{\lambda}_{1}=\lambda_{1}-\frac{\varepsilon}{p} \in\left(0, \frac{2}{\alpha}\right) \cap(0, \lambda)\left(0<\hat{\lambda}_{2}=\lambda_{2}+\frac{\varepsilon}{p}=\lambda-\hat{\lambda}_{1}<\lambda\right),
$$

we find

$$
\begin{aligned}
\widetilde{I} & =\sum_{n=1}^{\infty}\left[n^{\beta \hat{\lambda}_{2}} \sum_{m=1}^{\infty} \frac{1}{\left(m^{\alpha}+n^{\beta}\right)^{\lambda}} m^{\alpha \hat{\lambda}_{1}-1}\right] n^{-\beta \varepsilon-1} \\
& =\frac{1}{\alpha} \sum_{n=1}^{\infty} \omega\left(\hat{\lambda}_{1}, n\right) n^{-\beta \varepsilon-1}>\frac{1}{\alpha} k_{\lambda}\left(\hat{\lambda}_{1}\right) \sum_{n=1}^{\infty}\left(1-O\left(\frac{1}{n^{\beta \hat{\lambda}_{1}}}\right)\right) n^{-\beta \varepsilon-1} \\
& =\frac{1}{\alpha} k_{\lambda}\left(\hat{\lambda}_{1}\right)\left(\sum_{n=1}^{\infty} n^{-\beta \varepsilon-1}-\sum_{n=1}^{\infty} \frac{1}{O\left(n^{\beta\left(\lambda_{1}+\frac{\varepsilon}{q}\right)+1}\right)}\right) \\
& >\frac{1}{\alpha} k_{\lambda}\left(\hat{\lambda}_{1}\right)\left(\int_{1}^{\infty} x^{-\beta \varepsilon-1} d x-O(1)\right) \\
& =\frac{1}{\varepsilon \alpha \beta} k_{\lambda}\left(\hat{\lambda}_{1}\right)(1-\varepsilon \beta O(1)) .
\end{aligned}
$$

In virtue of the above results, we have

$$
\frac{1}{\alpha \beta} B\left(\lambda_{1}-\frac{\varepsilon}{p}, \lambda_{2}+\frac{\varepsilon}{p}\right)(1-\varepsilon \beta O(1))<\varepsilon \widetilde{I}<M\left(\frac{1}{\alpha}+\varepsilon\right)^{\frac{1}{p}}\left(\frac{1}{\beta}+\varepsilon\right)^{\frac{1}{q}} .
$$

For $\varepsilon \rightarrow 0^{+}$, in view of the continuity of the beta function, we get

$$
K_{\lambda}\left(\lambda_{1}\right)=\frac{1}{\alpha^{1 / q} \beta^{1 / p}} B\left(\lambda_{1}, \lambda_{2}\right) \leq M .
$$


Hence, $M=K_{\lambda}\left(\lambda_{1}\right)$ is the best possible constant factor in (15). The Lemma 3 is proved.

Remark 2. Setting $\widetilde{\lambda}_{1}:=\frac{\lambda-\lambda_{2}}{p}+\frac{\lambda_{1}}{q}, \widetilde{\lambda}_{2}:=\frac{\lambda-\lambda_{1}}{q}+\frac{\lambda_{2}}{p}$, we find

$$
\widetilde{\lambda}_{1}+\widetilde{\lambda}_{2}=\frac{\lambda-\lambda_{2}}{p}+\frac{\lambda_{1}}{q}+\frac{\lambda-\lambda_{1}}{q}+\frac{\lambda_{2}}{p}=\frac{\lambda}{p}+\frac{\lambda}{q}=\lambda,
$$

and thus we can rewrite inequality (13) as

$$
\begin{aligned}
I= & \sum_{n=1}^{\infty} \sum_{m=1}^{\infty} \frac{a_{m} b_{n}}{\left(m^{\alpha}+n^{\beta}\right)^{\lambda}}<\left(\frac{1}{\beta} k_{\lambda}\left(\lambda_{2}\right)\right)^{\frac{1}{p}}\left(\frac{1}{\alpha} k_{\lambda}\left(\lambda_{1}\right)\right)^{\frac{1}{q}} \\
& \times\left[\sum_{m=1}^{\infty} m^{p\left(1-\alpha \tilde{\lambda}_{1}\right)-1} a_{m}^{p}\right]^{\frac{1}{p}}\left[\sum_{n=1}^{\infty} n^{q\left(1-\beta \widetilde{\lambda}_{2}\right)-1} b_{n}^{q}\right]^{\frac{1}{q}}
\end{aligned}
$$

Lemma 4. If inequality (17) has the best possible constant factor $\left(\frac{1}{\beta} k_{\lambda}\left(\lambda_{2}\right)\right)^{\frac{1}{p}}\left(\frac{1}{\alpha} k_{\lambda}\left(\lambda_{1}\right)\right)^{\frac{1}{q}}$ for various parameters, then $\lambda=\lambda_{1}+\lambda_{2}$.

Proof. Note that

$$
\begin{gathered}
\widetilde{\lambda}_{1}=\frac{\lambda-\lambda_{2}}{p}+\frac{\lambda_{1}}{q}>0, \widetilde{\lambda}_{1}<\frac{\lambda}{p}+\frac{\lambda}{q}=\lambda, \\
0<\widetilde{\lambda}_{2}=\lambda-\widetilde{\lambda}_{1}<\lambda .
\end{gathered}
$$

Hence, we have

$$
k_{\lambda}\left(\widetilde{\lambda}_{1}\right)=B\left(\widetilde{\lambda}_{1}, \widetilde{\lambda}_{2}\right) \in \mathrm{R}_{+}=(0, \infty)
$$

If the constant factor $\left(\frac{1}{\beta} k_{\lambda}\left(\lambda_{2}\right)\right)^{\frac{1}{p}}\left(\frac{1}{\alpha} k_{\lambda}\left(\lambda_{1}\right)\right)^{\frac{1}{q}}$ in (17) is the best possible, then in view of (15), we have

$$
\left(\frac{1}{\beta} k_{\lambda}\left(\lambda_{2}\right)\right)^{\frac{1}{p}}\left(\frac{1}{\alpha} k_{\lambda}\left(\lambda_{1}\right)\right)^{\frac{1}{q}} \leq K_{\lambda}\left(\widetilde{\lambda}_{1}\right)
$$

namely, $k_{\lambda}^{\frac{1}{p}}\left(\lambda_{2}\right) k_{\lambda}^{\frac{1}{q}}\left(\lambda_{1}\right) \leq k_{\lambda}\left(\widetilde{\lambda}_{1}\right)$.

Applying Hölder's inequality (see [27]), we obtain

$$
\begin{aligned}
& k_{\lambda}\left(\widetilde{\lambda}_{1}\right)=k_{\lambda}\left(\frac{\lambda-\lambda_{2}}{p}+\frac{\lambda_{1}}{q}\right) \\
& =\int_{0}^{\infty} \frac{1}{(1+u)^{\lambda}} u^{\frac{\lambda-\lambda_{2}}{p}+\frac{\lambda_{1}}{q}-1} d u=\int_{0}^{\infty} \frac{1}{(1+u)^{\lambda}}\left(u^{\frac{\lambda-\lambda_{2}-1}{p}}\right)\left(u^{\frac{\lambda_{1}-1}{q}}\right) d u \\
& \leq\left[\int_{0}^{\infty} \frac{1}{(1+u)^{\lambda}} u^{\lambda-\lambda_{2}-1} d u\right]^{\frac{1}{p}}\left[\int_{0}^{\infty} \frac{1}{(1+u)^{\lambda}} u^{\lambda_{1}-1} d u\right]^{\frac{1}{q}} \\
& =\left[\int_{0}^{\infty} \frac{1}{(1+v)^{\lambda}} v^{\lambda_{2}-1} d v\right]^{\frac{1}{p}}\left[\int_{0}^{\infty} \frac{1}{(1+u)^{\lambda}} u^{\lambda_{1}-1} d u\right]^{\frac{1}{q}} \\
& =k_{\lambda}^{\frac{1}{p}}\left(\lambda_{2}\right) k_{\lambda}^{\frac{1}{q}}\left(\lambda_{1}\right) .
\end{aligned}
$$

Hence $k_{\lambda}^{\frac{1}{p}}\left(\lambda_{2}\right) k_{\lambda}^{\frac{1}{q}}\left(\lambda_{1}\right)=k_{\lambda}\left(\widetilde{\lambda}_{1}\right)$, which implies that (18) keeps the form of equality.

We observe that (18) keeps the form of equality if and only if there exist constants $A$ and $B$ such that they are not both zero and (see [27]) $A u^{\lambda-\lambda_{2}-1}=B u^{\lambda_{1}-1}$ a.e. in $\mathrm{R}_{+}$.

Assuming that $A \neq 0$, we have $u^{\lambda-\lambda_{2}-\lambda_{1}}=\frac{B}{A}$ a.e. in $\mathrm{R}_{+}$.

Hence $\lambda-\lambda_{2}-\lambda_{1}=0$, namely, $\lambda=\lambda_{1}+\lambda_{2}$. This completes the proof of Lemma 4 . 


\section{Main Results}

Theorem 1. Inequality (13) is equivalent to the following

$$
\begin{gathered}
J:=\left\{\sum_{n=1}^{\infty} n^{p \beta\left(\frac{\lambda-\lambda_{1}}{q}+\frac{\lambda_{2}}{p}\right)-1}\left[\sum_{m=1}^{\infty} \frac{1}{\left(m^{\alpha}+n^{\beta}\right)^{\lambda}} a_{m}\right]^{p}\right\}^{\frac{1}{p}} \\
<\left(\frac{1}{\beta} k_{\lambda}\left(\lambda_{2}\right)\right)^{\frac{1}{p}}\left(\frac{1}{\alpha} k_{\lambda}\left(\lambda_{1}\right)\right)^{\frac{1}{q}}\left\{\sum_{m=1}^{\infty} m^{p\left[1-\alpha\left(\frac{\lambda-\lambda_{2}}{p}+\frac{\lambda_{1}}{q}\right)\right]-1} a_{m}^{p}\right\}^{\frac{1}{p}} .
\end{gathered}
$$

If the constant factor in (13) is the best possible, then so is the constant factor in (19).

Proof. Firstly, we show that inequality (19) implies inequality (13). By using Hölder's inequality (see [27]), we have

$$
I=\sum_{n=1}^{\infty}\left[n^{\frac{-1}{p}+\beta\left(\frac{\lambda-\lambda_{1}}{q}+\frac{\lambda_{2}}{p}\right)} \sum_{m=1}^{\infty} \frac{1}{\left(m^{\alpha}+n^{\beta}\right)^{\lambda}} a_{m}\right]\left[n^{\frac{1}{p}-\beta\left(\frac{\lambda-\lambda_{1}}{q}+\frac{\lambda_{2}}{p}\right)} b_{n}\right] \leq J\left\{\sum_{n=1}^{\infty} n^{q\left[1-\beta\left(\frac{\lambda-\lambda_{1}}{q}+\frac{\lambda_{2}}{p}\right)\right]-1} b_{n}^{q}\right\}^{\frac{1}{q}} .
$$

Then from inequality (19), we obtain inequality (13). we set

Next, we show that inequality (13) implies inequality (19). Assuming that inequality (13) is valid,

$$
b_{n}:=n^{p \beta\left(\frac{\lambda-\lambda_{1}}{q}+\frac{\lambda_{2}}{p}\right)-1}\left[\sum_{m=1}^{\infty} \frac{1}{\left(m^{\alpha}+n^{\beta}\right)^{\lambda}} a_{m}^{p-1}, n \in \mathrm{N} .\right.
$$

If $J=0$, then inequality (19) is naturally valid; if $J=\infty$, then it is impossible to make inequality (19) valid, namely, $J<\infty$. Suppose that $0<J<\infty$. By inequality (13), we have

$$
\begin{aligned}
& \sum_{n=1}^{\infty} n^{q\left[1-\beta\left(\frac{\lambda-\lambda_{1}}{q}+\frac{\lambda_{2}}{p}\right)\right]-1} b_{n}^{q}=J^{p}=I<\left(\frac{1}{\beta} k_{\lambda}\left(\lambda_{2}\right)\right)^{\frac{1}{p}}\left(\frac{1}{\alpha} k_{\lambda}\left(\lambda_{1}\right)\right)^{\frac{1}{q}} \\
& \times\left\{\sum_{m=1}^{\infty} m^{p\left[1-\alpha\left(\frac{\lambda-\lambda_{2}}{p}+\frac{\lambda_{1}}{q}\right)\right]-1} a_{m}^{p}\right\}^{\frac{1}{p}}\left\{\sum_{n=1}^{\infty} n^{q\left[1-\beta\left(\frac{\lambda-\lambda_{1}}{q}+\frac{\lambda_{2}}{p}\right)\right]-1} b_{n}^{q}\right\}^{\frac{1}{q}}, \\
& J=\left\{\sum_{n=1}^{\infty} n^{q\left[1-\beta\left(\frac{\lambda-\lambda_{1}}{q}+\frac{\lambda_{2}}{p}\right)\right]-1} b_{n}^{q}\right\}^{\frac{1}{p}}<\left(\frac{1}{\beta} k_{\lambda}\left(\lambda_{2}\right)\right)^{\frac{1}{p}}\left(\frac{1}{\alpha} k_{\lambda}\left(\lambda_{1}\right)\right)^{\frac{1}{q}} \\
& \times\left\{\sum_{m=1}^{\infty} m^{p\left[1-\alpha\left(\frac{\lambda-\lambda_{2}}{p}+\frac{\lambda_{1}}{q}\right)\right]-1} a_{m}^{p}\right\}^{\frac{1}{p}}
\end{aligned}
$$

which leads to inequality (19). Hence, inequality (13) is equivalent to inequality (19).

By means of the above obtained result, we can conclude that if the constant factor in (13) is the best possible, then so is the constant factor in (19). Otherwise, if there exists a constant $M\left(M<\left(\frac{1}{\beta} k_{\lambda}\left(\lambda_{2}\right)\right)^{\frac{1}{p}}\left(\frac{1}{\alpha} k_{\lambda}\left(\lambda_{1}\right)\right)^{\frac{1}{q}}\right)$ such that (19) is valid by replacing $\left(\frac{1}{\beta} k_{\lambda}\left(\lambda_{2}\right)\right)^{\frac{1}{p}}\left(\frac{1}{\alpha} k_{\lambda}\left(\lambda_{1}\right)\right)^{\frac{1}{q}}$ by $M$, then by (20), we would reach a contradiction that the constant factor $\left(\frac{1}{\beta} k_{\lambda}\left(\lambda_{2}\right)\right)^{\frac{1}{p}}\left(\frac{1}{\alpha} k_{\lambda}\left(\lambda_{1}\right)\right)^{\frac{1}{q}}$ in (13) is not the best possible. The proof of Theorem 1 is complete.

Theorem 2. The following statements (i), (ii), (iii) and (iv) are equivalent:

(i) $\operatorname{Both} k_{\lambda}^{\frac{1}{p}}\left(\lambda_{2}\right) k_{\lambda}^{\frac{1}{q}}\left(\lambda_{1}\right)$ and $k_{\lambda}\left(\frac{\lambda-\lambda_{2}}{p}+\frac{\lambda_{1}}{q}\right)$ are independent of $p, q$;

(ii) $k_{\lambda}^{\frac{1}{p}}\left(\lambda_{2}\right) k_{\lambda}^{\frac{1}{q}}\left(\lambda_{1}\right)$ is expressible as a single integral

$$
k_{\lambda}\left(\frac{\lambda-\lambda_{2}}{p}+\frac{\lambda_{1}}{q}\right)=k_{\lambda}\left(\widetilde{\lambda}_{1}\right)=\int_{0}^{\infty} \frac{1}{(1+u)^{\lambda}} u^{\widetilde{\lambda}_{1}-1} d u
$$


(iii) $\left(\frac{1}{\beta} k_{\lambda}\left(\lambda_{2}\right)\right)^{\frac{1}{p}}\left(\frac{1}{\alpha} k_{\lambda}\left(\lambda_{1}\right)\right)^{\frac{1}{q}}$ in (13) is the best possible constant factor;

(iV) $\lambda=\lambda_{1}+\lambda_{2}$.

If the statement (iv) follows, namely, $\lambda=\lambda_{1}+\lambda_{2}$, then we have (15) and the following equivalent inequality with the best possible constant factor $K_{\lambda}\left(\lambda_{1}\right)$ :

$$
\left\{\sum_{n=1}^{\infty} n^{p \beta \lambda_{2}-1}\left[\sum_{m=1}^{\infty} \frac{1}{\left(m^{\alpha}+n^{\beta}\right)^{\lambda}} a_{m}\right]^{p \frac{1}{p}}<K_{\lambda}\left(\lambda_{1}\right)\left[\sum_{m=1}^{\infty} m^{p\left(1-\alpha \lambda_{1}\right)-1} a_{m}^{p}\right]^{\frac{1}{p}}\right.
$$

Proof. (i) $\Rightarrow$ (ii). By (i), in view of the continuity of the Beta function, we have

$$
\begin{gathered}
k_{\lambda}^{\frac{1}{p}}\left(\lambda_{2}\right) k_{\lambda}^{\frac{1}{q}}\left(\lambda_{1}\right)=\lim _{p \rightarrow \infty} \lim _{q \rightarrow 1^{+}} k_{\lambda}^{\frac{1}{p}}\left(\lambda_{2}\right) k_{\lambda}^{\frac{1}{q}}\left(\lambda_{1}\right)=k_{\lambda}\left(\lambda_{1}\right), \\
k_{\lambda}\left(\frac{\lambda-\lambda_{2}}{p}+\frac{\lambda_{1}}{q}\right)=\lim _{p \rightarrow \infty} \lim _{q \rightarrow 1^{+}} k_{\lambda}\left(\widetilde{\lambda}_{1}\right)=\lim _{p \rightarrow \infty} \lim _{q \rightarrow 1^{+}} B\left(\widetilde{\lambda}_{1}, \lambda-\widetilde{\lambda}_{1}\right) \\
=B\left(\lambda_{1}, \lambda-\lambda_{1}\right)=k_{\lambda}\left(\lambda_{1}\right),
\end{gathered}
$$

namely, $k_{\lambda}^{\frac{1}{p}}\left(\lambda_{2}\right) k_{\lambda}^{\frac{1}{q}}\left(\lambda_{1}\right)$ is expressible as a single integral

$$
k_{\lambda}\left(\widetilde{\lambda}_{1}\right)=\int_{0}^{\infty} \frac{1}{(1+u)^{\lambda}} u^{\tilde{\lambda}_{1}-1} d u
$$

(ii) $\Rightarrow$ (iv). If $k_{\lambda}^{\frac{1}{p}}\left(\lambda_{2}\right) k_{\lambda}^{\frac{1}{q}}\left(\lambda_{1}\right)=k_{\lambda}\left(\widetilde{\lambda}_{1}\right)$, then (18) keeps the form of equality. In view of Lemma 4 , it follows that $\lambda=\lambda_{1}+\lambda_{2}$.

(iv) $\Rightarrow$ (i). If $\lambda=\lambda_{1}+\lambda_{2}$, then both $k_{\lambda}^{\frac{1}{p}}\left(\lambda_{2}\right) k_{\lambda}^{\frac{1}{q}}\left(\lambda_{1}\right)$ and $k_{\lambda}\left(\frac{\lambda-\lambda_{2}}{p}+\frac{\lambda_{1}}{q}\right)$ are equal to $k_{\lambda}\left(\lambda_{1}\right)$, which are independent of $p, q$. Hence, it follows that (i) $\Leftrightarrow$ (ii) $\Leftrightarrow$ (iv).

(iii) $\Rightarrow$ (iv). By Lemma 4 , we have $\lambda=\lambda_{1}+\lambda_{2}$.

(iv) $\Rightarrow$ (iii). By Lemma 3, for $\lambda=\lambda_{1}+\lambda_{2}$,

$$
\left(\frac{1}{\beta} k_{\lambda}\left(\lambda_{2}\right)\right)^{\frac{1}{p}}\left(\frac{1}{\alpha} k_{\lambda}\left(\lambda_{1}\right)\right)^{\frac{1}{q}}\left(=K_{\lambda}\left(\lambda_{1}\right)\right)
$$

is the best possible constant factor in (13). Therefore, we have (iii) $\Leftrightarrow$ (iv).

Hence, we conclude that the statements (i), (ii), (iii) and (iv) are equivalent. This completes the proof of Theorem 2.

\section{Operator Expressions and Some Particular Cases}

We choose functions

$$
\varphi(m):=m^{p\left[1-\alpha\left(\frac{\lambda-\lambda_{2}}{p}+\frac{\lambda_{1}}{q}\right)\right]-1}, \psi(n):=n^{q\left[1-\beta\left(\frac{\lambda-\lambda_{1}}{q}+\frac{\lambda_{2}}{p}\right)\right]-1},
$$

wherefrom,

$$
\psi^{1-p}(n)=n^{p \beta\left(\frac{\lambda-\lambda_{1}}{q}+\frac{\lambda_{2}}{p}\right)-1}(m, n \in \mathbf{N}) .
$$

Define the following real normed spaces: 


$$
\begin{aligned}
& l_{p, \varphi}:=\left\{a=\left\{a_{m}\right\}_{m=1}^{\infty} ;\|a\|_{p, \varphi}:=\left(\sum_{m=1}^{\infty} \varphi(m)\left|a_{m}\right|^{p}\right)^{\frac{1}{p}}<\infty\right\}, \\
& l_{q, \psi}:=\left\{b=\left\{b_{n}\right\}_{n=1}^{\infty} ;\|b\|_{q, \psi}:=\left(\sum_{n=1}^{\infty} \psi(n)\left|b_{n}\right|^{q}\right)^{\frac{1}{q}}<\infty\right\}, \\
& l_{p, \psi^{1-p}}:=\left\{c=\left\{c_{n}\right\}_{n=1}^{\infty} ;\|c\|_{p, \psi^{1-p}}:=\left(\sum_{n=1}^{\infty} \psi^{1-p}(n)\left|c_{n}\right|^{p}\right)^{\frac{1}{p}}<\infty\right\} .
\end{aligned}
$$

Let $a \in l_{p, \varphi}$, and let

$$
c=\left\{c_{n}\right\}_{n=1}^{\infty}, c_{n}:=\sum_{m=1}^{\infty} \frac{1}{\left(m^{\alpha}+n^{\beta}\right)^{\lambda}} a_{m}, n \in \mathrm{N} .
$$

Then, we can rewrite (19) as follows:

$$
\|c\|_{p, \psi^{1-p}}<\left(\frac{1}{\beta} k_{\lambda}\left(\lambda_{2}\right)\right)^{\frac{1}{p}}\left(\frac{1}{\alpha} k_{\lambda}\left(\lambda_{1}\right)\right)^{\frac{1}{\varphi}}\|a\|_{p, \varphi}<\infty,
$$

that is, $c \in l_{p, \psi^{1-p}}$.

Definition 1. Define an extended Hardy-Hilbert's operator $T: l_{p, \varphi} \rightarrow l_{p, \psi^{1-p}}$ as follows:

For any $a \in l_{p, \varphi}$, there exists a unique representation $T a=c \in l_{p, \psi^{1-p}}$ satisfying, for any $n \in \mathrm{N}$, $(T a)(n)=c_{n}$. Define the formal inner product of Ta and $b \in l_{q, \psi}$, and the norm of $T$ as follows:

$$
(T a, b):=\sum_{n=1}^{\infty}\left[\sum_{m=1}^{\infty} \frac{1}{\left(m^{\alpha}+n^{\beta}\right)^{\lambda}} a_{m}\right] b_{n},\|T\|:=\sup _{a(\neq \theta) \in l_{p, \varphi}} \frac{\|T a\|_{p, \psi^{1-p}}}{\|a\|_{p, \varphi}} .
$$

Then, by Theorems 1 and 2, we have

Theorem 3. If $a \in l_{p, \varphi}, b \in l_{q, \psi},\|a\|_{p, \varphi},\|b\|_{q, \psi}>0$, then we have the following inequalities:

$$
\begin{gathered}
(T a, b)<\left(\frac{1}{\beta} k_{\lambda}\left(\lambda_{2}\right)\right)^{\frac{1}{p}}\left(\frac{1}{\alpha} k_{\lambda}\left(\lambda_{1}\right)\right)^{\frac{1}{q}}\|a\|_{p, \varphi}\|b\|_{q, \psi}, \\
\|T a\|_{p, \psi^{1-p}}<\left(\frac{1}{\beta} k_{\lambda}\left(\lambda_{2}\right)\right)^{\frac{1}{p}}\left(\frac{1}{\alpha} k_{\lambda}\left(\lambda_{1}\right)\right)^{\frac{1}{q}}\|a\|_{p, \varphi} .
\end{gathered}
$$

Moreover, $\lambda_{1}+\lambda_{2}=\lambda$ if, and only if, the constant factor

$$
\left(\frac{1}{\beta} k_{\lambda}\left(\lambda_{2}\right)\right)^{\frac{1}{p}}\left(\frac{1}{\alpha} k_{\lambda}\left(\lambda_{1}\right)\right)^{\frac{1}{q}}
$$

in (22) and (23) is the best possible, namely,

$$
\|T\|=K_{\lambda}\left(\lambda_{1}\right)=\frac{1}{\alpha^{1 / q} \beta^{1 / p}} B\left(\lambda_{1}, \lambda_{2}\right) .
$$

Remark 3. (i) Taking $\alpha=\beta=1, \lambda_{1}, \lambda_{2} \in(0,2]\left(\lambda_{1}+\lambda_{2}=\lambda \in(0,4]\right)$ in (15) and (21), we obtain inequality (3) and the following inequality with the best possible constant factor $B\left(\lambda_{1}, \lambda_{2}\right)$ :

$$
\left\{\sum_{n=1}^{\infty} n^{p \lambda_{2}-1}\left[\sum_{m=1}^{\infty} \frac{1}{(m+n)^{\lambda}} a_{m}\right]^{p}\right\}^{\frac{1}{p}}<B\left(\lambda_{1}, \lambda_{2}\right)\left[\sum_{m=1}^{\infty} m^{p\left(1-\lambda_{1}\right)-1} a_{m}^{p}\right]^{\frac{1}{p}} .
$$

Hence, inequalities (13) and (15) are new extensions of inequality (1). 
(ii) Taking $\alpha=\beta=\frac{1}{2}, \lambda_{1}, \lambda_{2} \in(0,4]\left(\lambda_{1}+\lambda_{2}=\lambda \in(0,6]\right)$ in (15) and (21), we get the following inequality with the best possible constant factor $2 B\left(\lambda_{1}, \lambda_{2}\right)$ :

$$
\begin{gathered}
\sum_{n=1}^{\infty} \sum_{m=1}^{\infty} \frac{a_{m} b_{n}}{(\sqrt{m}+\sqrt{n})^{\lambda}}<2 B\left(\lambda_{1}, \lambda_{2}\right)\left[\sum_{m=1}^{\infty} m^{p\left(1-\frac{\lambda_{1}}{2}\right)-1} a_{m}^{p}\right]^{\frac{1}{p}}\left[\sum_{n=1}^{\infty} n^{q\left(1-\frac{\lambda_{2}}{2}\right)-1} b_{n}^{q}\right]^{\frac{1}{q}}, \\
\left\{\sum_{n=1}^{\infty} n^{\frac{p \lambda_{2}}{2}-1}\left[\sum_{m=1}^{\infty} \frac{1}{(\sqrt{m}+\sqrt{n})^{\lambda}} a_{m}\right]^{p}\right\}^{\frac{1}{p}}<2 B\left(\lambda_{1}, \lambda_{2}\right)\left[\sum_{m=1}^{\infty} m^{p\left(1-\frac{\lambda_{1}}{2}\right)-1} a_{m}^{p}\right]^{\frac{1}{p}} .
\end{gathered}
$$

(iii) Taking $\alpha=\beta=\frac{2}{3}, \lambda_{1}, \lambda_{2} \in(0,3]\left(\lambda_{1}+\lambda_{2}=\lambda \in(0,6]\right)$ in (15) and (21), we derive the following inequality with the best possible constant factor $\frac{3}{2} B\left(\lambda_{1}, \lambda_{2}\right)$ :

$$
\begin{gathered}
\sum_{n=1}^{\infty} \sum_{m=1}^{\infty} \frac{a_{m} b_{n}}{\left(\sqrt[3]{m^{2}}+\sqrt[3]{n^{2}}\right)^{\lambda}}<\frac{3}{2} B\left(\lambda_{1}, \lambda_{2}\right)\left[\sum_{m=1}^{\infty} m^{p\left(1-\frac{2 \lambda_{1}}{3}\right)-1} a_{m}^{p}\right]^{\frac{1}{p}}\left[\sum_{n=1}^{\infty} n^{q\left(1-\frac{2 \lambda_{2}}{3}\right)-1} b_{n}^{q}\right]^{\frac{1}{q}}, \\
\left\{\sum_{n=1}^{\infty} n^{\frac{2 p \lambda_{2}}{3}-1}\left[\sum_{m=1}^{\infty} \frac{1}{\left(\sqrt[3]{m^{2}}+\sqrt[3]{n^{2}}\right)^{\lambda}} a_{m}\right]^{p}\right\}^{\frac{1}{p}}<\frac{3}{2} B\left(\lambda_{1}, \lambda_{2}\right)\left[\sum_{m=1}^{\infty} m^{p\left(1-\frac{2 \lambda_{1}}{3}\right)-1} a_{m}^{p}\right]^{\frac{1}{p}}
\end{gathered}
$$

\section{Conclusions}

We first provided a brief survey on the study of Hardy-Hilbert's inequality, and then we stated the main results, new extensions of Hardy-Hilbert's inequality, in Lemma 2 and Theorem 1, respectively. For further study on the obtained inequalities, the equivalent statements of the best possible constant factor related to several parameters are given in Theorem 2; the operator expressions of the extended Hardy-Hilbert's inequality are established in Theorem 3. It is worth noting that the extended Hardy-Hilbert's inequality (13) obtained in this paper differs from the inequality (9) that appeared in [26], since inequalities (9) and (13) contain different kernels of $\frac{1}{m^{\lambda}+n^{\lambda}}$ and $\frac{1}{\left(m^{\alpha}+n^{\beta}\right)^{\lambda}}$. We also note that the two kinds of kernels have a similar form; this prompts us to consider the meaningful problem of how to establish a unified extension of inequalities (9) and (13) in a subsequent study.

Author Contributions: B.Y. carried out the mathematical studies and drafted the manuscript. S.W. and Q.C. participated in the design of the study and performed the numerical analysis. All authors contributed equally and significantly in this paper. All authors have read and agreed to the published version of the manuscript.

Funding: This work is supported by the National Natural Science Foundation (No. 61772140), and the Science and Technology Planning Project Item of Guangzhou City (No. 201707010229).

Acknowledgments: The authors are grateful to the reviewers for their valuable comments and suggestions to improve the quality of the manuscript.

Conflicts of Interest: The authors declare no conflict of interest.

\section{References}

1. Hardy, G.H.; Littlewood, J.E.; Polya, G. Inequalities; Cambridge University Press: Cambridge, UK, 1934.

2. Yang, B.C. On a generalization of Hilbert double series theorem. J. Nanjing Univ. Math. 2001, 18, 145-152.

3. Krnić, M.; Pečarić, J. Extension of Hilbert's inequality. J. Math. Anal. Appl. 2006, 324, 150-160. [CrossRef]

4. Adiyasuren, V.; Batbold, T.; Azar, L.E. A new discrete Hilbert-type inequality involving partial sums. J. Inequal. Appl. 2019, 2019, 127. [CrossRef]

5. Yang, B.C. The Norm of Operator and Hilbert-type Inequalities; Science Press: Beijing, China, 2009.

6. Krnić, M.; Pečarić, J. General Hilbert's and Hardy's inequalities. Math. Inequal. Appl. 2005, 8, $29-51$. [CrossRef] 
7. Perić, I.; Vuković, P. Multiple Hilbert's type inequalities with a homogeneous kernel. Banach J. Math. Anal. 2011, 5, 33-43. [CrossRef]

8. Huang, Q.L. A new extension of Hardy-Hilbert-type inequality. J. Inequal. Appl. 2015, 2015, 397. [CrossRef]

9. He, B.; Wang, Q. A multiple Hilbert-type discrete inequality with a new kernel and best possible constant factor. J. Math. Anal. Appl. 2015, 431, 889-902. [CrossRef]

10. Xu, J.S. Hardy-Hilbert's inequalities with two parameters. Adv. Math. 2007, 36, 63-76.

11. Xie, Z.T.; Zeng, Z.; Sun, Y.F. A new Hilbert-type inequality with the homogeneous kernel of degree -2 . Adv. Appl. Math. Sci. 2013, 12, 391-401.

12. Zheng, Z.; Gandhi, R.R.; Xie, Z.T. A new Hilbert-type inequality with the homogeneous kernel of degree -2 and with the integral. Bull. Math. Sci. Appl. 2014, 7, 9-17. [CrossRef]

13. Xin, D.M. A Hilbert-type integral inequality with the homogeneous kernel of zero degree. Math. Theory Appl. 2010, 30, 70-74.

14. Azar, L.E. The connection between Hilbert and Hardy inequalities. J. Inequal. Appl. 2013, 2013, 452. [CrossRef]

15. Adiyasuren, V.; Batbold, T.; Krnić, M. Hilbert-type inequalities involving differential operators, the best constants and applications. Math. Inequal. Appl. 2015, 18, 111-124. [CrossRef]

16. Rassias, M.T.; Yang, B.C. On half-discrete Hilbert's inequality. Appl. Math. Comput. 2013, 220, 75-93. [CrossRef]

17. Yang, B.C.; Krnić, M. A half-discrete Hilbert-type inequality with a general homogeneous kernel of degree 0 . J. Math. Inequal. 2012, 6, 401-417.

18. Rassias, M.T.; Yang, B.C. A multidimensional half-Discrete Hilbert-Type inequality and the Riemann zeta function. Appl. Math. Comput. 2013, 225, 263-277. [CrossRef]

19. Rassias, M.T.; Yang, B.C. On a multidimensional half-discrete Hilbert-Type inequality related to the hyperbolic cotangent function. Appl. Math. Comput. 2014, 242, 800-813. [CrossRef]

20. Yang, B.C.; Debnath, L. Half-Discrete Hilbert-Type Inequalitiesl; World Scientific Publishing: Singapore, 2014.

21. Yang, B.C.; Wu, S.H.; Wang, A.Z. On a reverse half-discrete Hardy-Hilbert's inequality with parameters. Mathematics 2019, 7, 1054. [CrossRef]

22. Liao, J.Q.; Wu, S.H.; Yang, B.C. On a new half-discrete Hilbert-type inequality involving the variable upper limit integral and partial sums. Mathematics 2020, 8, 229. [CrossRef]

23. Hong, Y.; Wen, Y. A necessary and sufficient condition of that Hilbert type series inequality with homogeneous kernel has the best constant factor. Ann. Math. 2016, 37, 329-336.

24. Yang, B.C.; Wu, S.H.; Chen, Q. On an extended Hardy-Littlewood-Polya's inequality. AIMS Math. 2020, 5, 1550-1561. [CrossRef]

25. Yang, B.C.; Wu, S.H.; Wang, A.Z. A new Hilbert-type inequality with positive homogeneous kernel and its equivalent forms. Symmetry 2020, 12, 342. [CrossRef]

26. Yang, B.C.; Wu, S.H.; Liao, J.Q. On a new extended Hardy-Hilbert's inequality with parameters. Mathematics 2020, 8, 73. [CrossRef]

27. Kuang, J.C. Applied Inequalitie; Shangdong Science and Technology Press: Jinan, China, 2004.

(C) 2020 by the authors. Licensee MDPI, Basel, Switzerland. This article is an open access article distributed under the terms and conditions of the Creative Commons Attribution (CC BY) license (http://creativecommons.org/licenses/by/4.0/). 